\title{
Branched-Chain Amino Acid Carbon and Nitrogen Arteriovenous Concentration Differences Across the Ovine Fetal Hindlimb
}

\author{
EDWARD A. LIECHTY, MARK J. POLAK, AND JAMES A. LEMONS \\ Department of Pediatrics, West Virginia University, School of Medicine, Morgantown, West Virginia 26506 and \\ Department of Pediatrics, Indiana University, School of Medicine, Indianapolis, Indiana 46223
}

\begin{abstract}
During fasting, branched-chain amino acids (BCAA) are thought to be major sources of nitrogen for myocyte synthesis of alanine (Ala) and glutamine (Gln), as well as possible sources of carbon skeleton for Gln synthesis. To study the relationships between Ala, GIn, and BCAA, we utilized the chronic fetal lamb preparation and measured arteriovenous concentration differences of Ala, Gln, BCAA, and branched-chain $\alpha$-keto acids across the fetal hindlimb. Studies were performed when the ewe was fed and repeated after 1 and 5 days of complete maternal fasting. Ala and Gln are released from fetal hindquarters during fasting (arteriovenous $-9.6 \pm 5$ and $-8.8 \pm 4.1$ $\mu \mathrm{mol} /$ liter), while arteriovenous differences for BCAA simultaneously increase by $65 \%$ as compared to the fed state. During fasting, total nitrogen exiting fetal hindlimb as Ala and GIn equals nitrogen entering as BCAA. Branched-chain $\alpha$-keto acids are released from fetal hindquarters during the fed state as well as after 1 day of fasting; at 5 days of fasting only keto-isovalerate had a net negative arteriovenous difference. In all cases, the release was much smaller than the entry of the corresponding amino acid, as determined by simultaneously measured arteriovenous concentration differences. These results indicate: 1) Ala and GIn are released from fetal hindlimb, as in postnatal animals, during fasting, shuttling nitrogen and carbon to liver and/or other tissues. 2) There is negligible movement of BCAA carbon as the branched-chain $\alpha$-keto acids from hindlimb to other tissues in the fetus. 3) BCAA carbon taken up is utilized within the hindquarters. It may be used for tissue synthesis, as an energy source, or possibly for Gln synthesis. (Pediatr Res 21: 44-48, 1987)
\end{abstract}

\section{Abbreviations}

KIC, $\alpha$-ketoisocaproate

KIV, $\alpha$-ketoisovalerate

KMV, $\alpha$-keto- $\beta$-methyl-valerate

$\mathrm{KC}, \alpha$-keto-caproic acid

ANOVA, analysis of variance

HSD, honestly significant difference

A-V, arteriovenous

ANCOVA, analysis of covariance
The fetus of a well-fed ewe relies primarily on glucose, glucosederived lactate, and free amino acids for energy substrate as well as substrate for tissue synthesis and growth (1). When glucose supply is adequate, glucose appears to be the most significant source of energy. However, when the ewe is fasted, glucose supply to the fetus is curtailed, obligating the fetus to diminish its metabolic rate or to make use of alternative substrates as sources of energy. Several studies have suggested that when so stressed, the ovine fetus may be capable of converting amino acids to glucose or lactate $(2,3)$. In either case, movement of amino acid carbon from protein stores to metabolically active tissues would be required. In addition, an efficient mechanism for transport of nitrogen from protein stores to the liver for urea formation would also be required.

It is clear that during maternal fasting, catabolism of amino acids by the fetus increases markedly (4). The total molar amount of amino acids acquired transplacentally by the fetus has been shown not to increase with maternal fasting (5); this implies that the ratio of amino acids catabolized to those used for tissue formation increases with maternal fasting.

In the adult organism, skeletal muscle represents a large repository of protein from which amino acids can be derived for gluconeogenesis. During fasting, alanine and glutamine move from peripheral tissues, principally skeletal muscle, to splanchnic tissues for gluconeogenic substrate. Muscle catabolism of the branched-chain amino acids also increases. It is controversial as to whether these amino acids are catabolized within skeletal muscle, or whether instead there may be significant interorgan transport of their transamination products, the branched-chain $\alpha$-keto acids.

We have previously demonstrated that, as in the adult, during maternal fasting fetal hindlimb exports alanine and glutamine in molar amounts greater than their representation in skeletal muscle protein (6). However, we also observed a simultaneous increase in the positive arteriovenous balance of the branchedchain amino acids, especially leucine, as compared to the fed state. The present study was undertaken to further delineate the relationships between branched-chain amino acids and glucose metabolism by the fetal hindlimb, and to examine the effect of maternal fasting on these relationships. A key aim of the study was to determine the extent to which the branched-chain amino acids are catabolized within the fetal hindlimb, or conversely, the significance of any interorgan transport of the branchedchain $\alpha$-keto acids.

\section{MATERIALS AND METHODS}

Received June 17, 1986; accepted August 12, 1986.
Correspondence and reprint requests Dr. E. A. Liechty, Department of Pediatrics. West Virginia University, School of Medicine, Morgantown, WV 26506.

This work was supported by National Institutes of Health Grant R01-HD19089. Biomedical Research Grant 2-S07-RR05433-22, and by the West Virginia University Medical Corporation.
Ewes of 115-120 days gestation were utilized for this study. Animal care was in strict compliance with National Institutes of Health guidelines within an accredited facility. Animals were 
allowed ad libitum access to food and water until $48 \mathrm{~h}$ prior to the surgical procedure, at which time fasting was instituted. Surgical procedures were performed aseptically under general anesthesia.

Surgical procedure. Anesthesia was accomplished by intravenous pentobarbital infusion. The pregnant horn of the uterus was exposed and a fetal hind limb brought through a $4-\mathrm{cm}$ uterine incision. The fetal femoral artery and vein were exposed and cannulated with Silastic (Dow-Corning, Medical Products, Midland, MI) catheters (ID $=0.030$ inches) and advanced to 10 and $6 \mathrm{~cm}$, respectively. Following closure of the uterus and abdomen, all catheters were tunneled beneath the ewe's skin and stored in a plastic pouch on the ewe's flank. The catheter placements consistently resulted in the venous catheter tip being situated below the renal veins, as confirmed in each animal at necropsy.

Study design. For a minimum of seven days postoperatively the ewe was fed ad libitum a standard diet for laboratory ruminants consisting of hay and pelletized oats. Blood samples were drawn on the 7th day, which represented the fed control study. All animals were allowed ad libitum access to food during the fed control study. Blood samples were drawn simultaneously from all catheters and processed for whole blood oxygen content, and whole blood concentrations of lactate, pyruvate, glucose, free amino acids, and the branched-chain $\alpha$-keto acids. Fasting was then begun, and blood sampling repeated at 24 and $120 \mathrm{~h}$ of complete maternal fasting. Eight animals that were each successfully studied in all three nutritional states are the basis for this report.

All blood sampling was done at the same time of day and in the same physical environment. Quadruplicate samples were drawn from each catheter for each substrate measured. Approximately $30 \mathrm{~min}$ elapsed between each of the four sampling sessions per study.

Analytical methods. Whole blood glucose was measured in 0.2 $\mathrm{ml}$ whole blood, after deproteinization with zinc sulfate and barium hydroxide, by the method of Raabo and Terkildsen (7), using a kit from Sigma (St. Louis, MO).

Lactate and pyruvate were determined by the method of Marbach and Weil (8) using a kit from Sigma. Intraassay coefficients of variation were less than $5 \%$ for both acids.

Oxygen content was determined on $20 \mu$ l of whole blood using a Lex- $\mathrm{O}_{2}$-Con (Lexington Instruments, Waltham, MA). Intraassay coefficient of variation was $3 \%$.

Amino acid concentrations were determined as described previously (6) using a Beckman $119 \mathrm{CL}$ amino acid analyzer. Sam- ples were stored at $-70^{\circ}$ until analysis. Intraassay coefficients of variations were less than $5 \%$ for each amino acid.

$\mathrm{KIC}, \mathrm{KIV}$, and KMV, were determined by a modification of the procedure described by Abumrad et al. (9). In brief, a 500- $\mu$ I sample of whole blood was pipeted into $1 \mathrm{ml}$ iced methanol to induce hemolysis and protein precipitation. The samples were kept at $0^{\circ}$ for $90 \mathrm{~min}$, followed by centrifugation at $3500 \mathrm{rpm}$ for $10 \mathrm{~min}$. Supernatant was removed and allowed to partially evaporate followed by derivatization with acidic 2,4-dinitrophenylhydrazine. Extraction of the derivatized acids was accomplished by adding $3 \mathrm{ml}$ cyclohexane:n-heptane:diethyl ether $(10: 1: 1)$ and shaking for 15 minutes. The organic layer was removed and evaporated to dryness, redissolved in $500 \mu \mathrm{l}$ methanol, and applied to a C-18 Sep-Pak (Waters, Milford, MA). The polar contaminants were eluted with $2 \mathrm{ml}$ water and discarded. The fraction of interest was then eluted from the Sep-Pak with 65:35 water:acetonitrile (v:v). This fraction was evaporated to dryness and redissolved in $150 \mu \mathrm{l}$ of methanol, which was used for the chromatographic separation and quantitation of the acids.

The high performance liquid chromatography system used consisted of dual Waters M45 pumps, a model 440 absorbance detector, and quantitation by a model 730 Data Module. Automated $10-\mu$ l injections were made onto a $5-\mu$ Resolve C-18 steel column. Mobile phase consisted of acetonitrile and $10 \mathrm{mM}$ $\mathrm{K}_{2} \mathrm{PO}_{4}, \mathrm{pH} 6.5$, with a gradient program from $10 \%$ acetonitrile to $40 \%$ acetonitrile. This gradient program was necessary to separate the KMV derivative from one of two KIC derivative isomers. Baseline separation was achieved for KIV and $\mathrm{KC} ; \mathrm{KIC}$ and KMV were seen as partially fused peaks, but sufficient separation was achieved for integration. Quantitation was by peak area relative to $\mathrm{KC}$, the internal standard. Intraassay coefficients of variation were $<10 \%$ for each of the compounds. Using spiked blood samples, quantitation was linear to $100 \mu \mathrm{m}$ concentrations.

Statistical methods. Data were analyzed by two-way ANOVA to determine significance between different nutritional states. Differences between specific means were evaluated by the Tukey's HSD method if the overall ANOVA F-statistic gave a $p<$ 0.05 for the effect of nutritional state. This method uses the studentized range and average cell sizes to compute a minimum range (HSD) which must be exceeded by the difference between a pair of means for that difference to be significant $(10,11)$. The HSDs computed for $p<0.05$ are included in Tables $1-4$, allowing comparison of any two of the three means. For A-V differences of the amino acids, the A-V difference for tyrosine (which did not vary with nutritional state) was used as a covariate, to account

Table 1. Changes in glucose, lactate, pyruvate, and oxygen A-V differences [mean (SEM) $n=8$ animals]

\begin{tabular}{|c|c|c|c|c|c|}
\hline & Fed & Fast 1 & Fast 5 & $p^{*}$ & $\mathrm{HSD}^{\dagger}$ \\
\hline \multicolumn{6}{|c|}{ Glucose $\mathrm{mM}$} \\
\hline Arterial & $1.03(0.05)$ & $0.789(0.044)$ & $0.613(0.040)$ & 0.001 & 0.147 \\
\hline$A-V$ & $0.181(0.03)$ & $0.130(0.024)$ & $0.050(0.028)$ & 0.001 & 0.115 \\
\hline \multicolumn{6}{|c|}{ Lactate $\mathrm{mM}$} \\
\hline Arterial & $0.602(0.03)$ & $0.681(0.074)$ & $1.00(0.20)$ & 0.026 & 0.360 \\
\hline$A-V$ & $-0.026(0.20)$ & $-0.022(0.18)$ & $-1.08(0.14)$ & NS & 0.201 \\
\hline Arterial & $36.4(1.7)$ & $37.9(1.5)$ & $41.3(3.2)$ & NS & 7.4 \\
\hline$A-V$ & $4.3(1.0)$ & $6.8(1.0)$ & $2.7(2.3)$ & NS & 5.2 \\
\hline \multicolumn{6}{|c|}{ Oxygen $\mathrm{mM}$} \\
\hline Arterial & $4.3(0.31)$ & $4.9(0.36)$ & $4.1(0.33)$ & NS & 1.1 \\
\hline$A-V$ & $0.80(0.07)$ & $1.1(0.11)$ & $0.80(0.07)$ & 0.05 & 0.30 \\
\hline
\end{tabular}

\footnotetext{
* $\mathrm{p}$ value determined by two-way ANOVA.
}

$\uparrow$ Tukey's HSD for critical range between means at $p<0.05$. 
Table 2. Effect of fasting on hindlimb A-V differences of alanine, glutamine, and tyrosine [mean (SEM) $\mu M /$ liter, $n=8$ animals]

\begin{tabular}{|c|c|c|c|c|c|}
\hline & Fed & Fast 1 & Fast 5 & $p^{*}$ & $\mathrm{HSD}+$ \\
\hline $\begin{array}{l}\text { Alanine } \\
\text { Arterial } \\
\text { A-V }\end{array}$ & $\begin{array}{l}310(7.9) \\
\quad 6.8(5.1)\end{array}$ & $\begin{array}{l}255(6) \\
0.8(3.9)\end{array}$ & $\begin{array}{c}252(13) \\
-9.6(5)\end{array}$ & $\begin{array}{r}<0.001 \\
0.013\end{array}$ & $\begin{array}{l}32 \\
11\end{array}$ \\
\hline $\begin{array}{l}\text { Glutamine } \\
\text { Arterial } \\
\mathrm{A}-\mathrm{V}\end{array}$ & $\begin{array}{r}287(8.9) \\
5(5.1)\end{array}$ & $\begin{array}{l}267(9.2) \\
1.6(3.8)\end{array}$ & $\begin{array}{c}249(9.9) \\
-8.8 \\
(4.1)\end{array}$ & $\begin{array}{r}<0.001 \\
0.037\end{array}$ & $\begin{array}{l}31 \\
10\end{array}$ \\
\hline $\begin{array}{c}\text { Tyrosine } \\
\text { Arterial } \\
\mathrm{A}-\mathrm{V}\end{array}$ & $\begin{array}{l}120(7) \\
3.9(2.9)\end{array}$ & $\begin{array}{c}100(3.9) \\
1.9(1.3)\end{array}$ & $\begin{array}{c}131(8.8) \\
2.6(2.6)\end{array}$ & $\begin{array}{c}<0.001 \\
\text { NS }\end{array}$ & $\begin{array}{c}23 \\
5.9\end{array}$ \\
\hline
\end{tabular}

* $p$ value determined by two-way ANOVA.

$\uparrow$ Tukey's HSD for critical range between means at $p<0.05$.

Table 3. Effect of fasting on hindlimb $A-V$ differences of branched chain amino acids [mean (SEM) $\mu M$ /liter, $n=8$ animals]

\begin{tabular}{lrrrrc}
\hline & Fed & \multicolumn{1}{c}{ Fast 1 } & Fast 5 & $p^{*}$ & HSD $\dagger$ \\
\hline Leucine & $14.6(2.4)$ & $15.3(2.4)$ & $21.5(4)$ & NS $\$$ & 7.0 \\
Isoleucine & $9.2(1.4)$ & $8.2(1.2)$ & $11.9(1.6)$ & NS $\$$ & 2.9 \\
Valine & $15.5(5.2)$ & $20.2(3.9)$ & $30.7(7.2)$ & NS $\$$ & 11 \\
\hline
\end{tabular}

$* p$ value determined by two-way ANOVA.

+ Tukey's HSD for critical range between means at $p<0.05$.

$\sharp p<0.05$ by ANCOVA, utilizing tyrosine $(\mathrm{A}-\mathrm{V})$ as covariate.

Table 4. Hindlimb arteriovenous differences of the branchedchain $\alpha$-keto acids [mean (SEM) uM/liter, $n=8$ animals]

\begin{tabular}{llcccc}
\hline & \multicolumn{1}{c}{ Fed } & Fast 1 & Fast 5 & $p^{*}$ & HSD $\dagger$ \\
\hline KIC & $-0.065(0.74)$ & $-1.17(0.89)$ & $0.137(1.6)$ & 0.17 & 3.7 \\
KIV & $-0.36(0.55)$ & $-0.56(0.55)$ & $-2.6(1.3)$ & 0.17 & 3.5 \\
KMV & $-0.29(0.47)$ & $-0.96(0.53)$ & $0.83(0.56)$ & 0.03 & 1.6 \\
\hline
\end{tabular}

* $p$ value determined by two-way ANOVA.

+ Tukey's HSD for critical range between means at $p<0.05$.

for small errors in sample preparation. Correlations were evaluated by standard simple or multiple regression techniques. All statistical analysis was done using a microcomputer based statistical package (Systat, Evanston, IL).

\section{RESULTS}

Glucose, lactate, and pyruvate balance. The arterial concentrations and $\mathrm{A}-\mathrm{V}$ differences for glucose, lactate, pyruvate, and oxygen are given in Table 1. Arterial glucose concentrations decreased significantly, from 1.03 to $0.613 \mathrm{mM}$ by the 5 th day of maternal fasting. A positive A-V difference for glucose across the fetal hindquarters was demonstrated, which also declined, from 0.181 to $0.050 \mathrm{mM}$ after fasting. This fasting value is much lower than that which we previously found $(0.099 \mathrm{mM})$ after a similar maternal fast. This difference cannot be explained on the basis of lower arterial glucose concentrations, as the fasted fetal glucose concentration of $0.613 \mathrm{mM}$ in the present study is actually slightly higher than that of $0.494 \mathrm{mM}$ found in our previous work.

Arterial lactate concentration increased during fasting, while pyruvate concentration remained stable. A consistent output of lactate from the hindlimb was observed. Although the mean lactate A-V difference increased by the 5th day of fasting, the increase was not statistically significant.

In contrast to lactate, pyruvate was taken up by the hindquarters. Fasting did not appear to affect the magnitude of the pyruvate arteriovenous difference.
Amino acid carbon balance. The arterial concentrations and A-V differences for alanine, glutamine, and tyrosine are shown in Table 2. Arterial concentrations of alanine and glutamine decreased during fasting. Both had a positive arteriovenous concentration difference during the fed state, which became negative, indicating venous efflux, during maternal fasting. The mean A$\mathrm{V}$ difference for tyrosine, an amino acid thought to be neither catabolized nor synthesized in skeletal muscle, remained slightly positive during fasting. This would indicate that little, if any, net hindlimb protein breakdown occurred during the fasted phase. This implies that the alanine and glutamine efflux represents net de novo synthesis of these two amino acids within the fetal hindlimb, as has been demonstrated in postnatal animals in vivo (12) as well as in cultured myocytes (13).

The arterial concentrations of the branched-chain amino acids increased greatly during the course of the maternal fast (Fig. 1). There was a very strong positive correlation between the arterial concentrations of isoleucine, valine, and leucine. $[(\mathrm{Val})=1.99$ (leu) $-16, r=96$; (lle) $=0.415$ (leu) $+11, r=0.90$.] Presumably this is reflective of their common metabolic pathway.

The A-V concentration differences for the branched-chain amino acids become increasingly positive during fasting (Table 3 ). In the case of valine, there was a doubling of the mean A-V difference. Using the A-V difference for tyrosine as a covariate, the changes in A-V difference by ANOVA were statistically significant for all three branched-chain amino acids. Because we did not measure blood flow, we cannot be sure that this indicates that the uptake of these amino acids increased.

The branched chain $\alpha$-keto acids were measured to determine the fate of the carbon skeleton of the branched-chain amino acid taken up by the hindlimb. Carbon skeleton of the branchedchain amino acids taken up by skeletal muscle could be utilized within the muscle, or the amino acid could be transaminated to the corresponding $\alpha$-keto acid which could subsequently be given

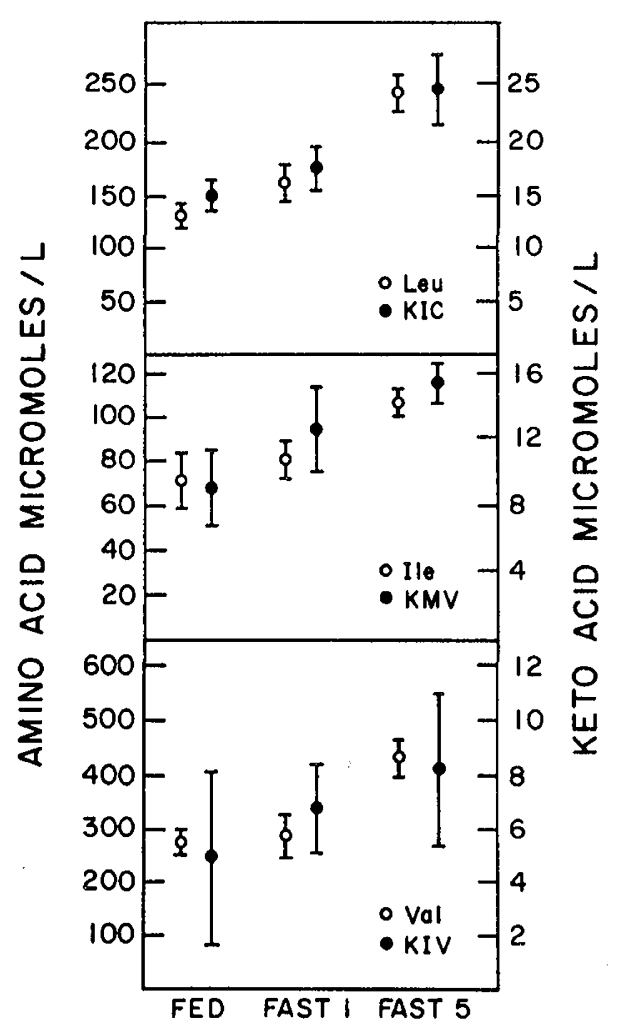

Fig. 1. Changes in arterial concentrations of the branched-chain amino acids and the corresponding change in arterial concentration of the $\alpha$-keto acids. Each point represents the mean \pm SEM of all animals $(n=8)$. 
off in the venous efflux. As can be seen in Figure 1, the arterial concentrations of all three $\alpha$-keto acids increased significantly over the course of maternal fasting. In general, the increased concentration paralleled the increased concentration of the corresponding amino acid, both in time and in percent change in concentration.

The A-V differences of the keto acids are seen in Table 4. As can be seen, the mean differences tended to be negative and to be relatively constant over the course of the fast. Only KMV was found to have a statistically significant difference over the fasting period, and this was isolated to the period between the 24-h fasted study and the 5-day fasted study. In all cases, however, the mean A-V molar concentration differences were very small in comparison to the simultaneous A-V molar concentration differences of the corresponding amino acids, implying near complete utilization of the carbon skeleton for energy substrate or synthesis of other molecules within the fetal hindlimb.

\section{DISCUSSION}

During the course of maternal fasting, the mammalian fetus becomes less reliant on transplacentally acquired glucose as a source of energy substrate. However, glucose utilization rates remain high and have been found to exceed transplacental uptake, implying a degree of glucogenesis during fasting. Also $25 \%$ of the lactate utilized by the fetus has been shown to arise from nonglucose precursors. These studies would suggest that the ovine fetus is capable of synthesizing lactate and possibly glucose from amino acid carbon skeleton when exogenous glucose supply is curtailed.

During a maternal fast, A-V concentration differences across the ovine fetal hindlimb increase. Simultaneously, carbon and nitrogen are exported from the hindlimb, as $\mathrm{A}-\mathrm{V}$ concentration differences of alanine and glutamine become negative. The results of the present study expand the thesis that alterations in fetal skeletal muscle metabolism during fasting are significant in conserving glucose as well as providing potential gluconeogenic substrate.

Arterial glucose levels, as well as A-V glucose differences, fall progressively during maternal fasting. The values obtained in the fed portion of the present study were comparable to those found in our previous study as well as that of Singh et al. (14). During fasting, however, the A-V glucose difference fell to very low levels, much lower than demonstrated in our previous study.

Lactate is released by fetal hindquarters during the fed and fasted states. Presumably, the lactate is derived from glucose uptake, although glycogen breakdown is a potential source, especially during the fasted state. Because the A-V difference for glucose was large relative to lactate $\mathrm{A}-\mathrm{V}$ difference during the fed state, a net glucose A-V difference of $0.168 \mathrm{mM}$ was observed. As the ewe was fasted, the A-V difference for glucose decreased to a much greater extent than did the lactate A-V difference. Therefore, the net glucose + lactate difference was $0.119 \mathrm{mM}$ after a 1-day fast, but was actually negative $(-0.04 \mathrm{mM})$, after the 5-day fast.

A small but consistent A-V difference was noted for pyruvate, the magnitude of which was not affected with fasting. Pyruvate would appear to be of negligible importance to the carbon and energy balance of the fetal hindlimb. However, the possibility that it exerts regulatory influence cannot be discounted.

Our data demonstrate a large positive $A-V$ concentration difference for each of the branched-chain amino acids by the fetal hindlimb, and demonstrate that this A-V difference increases with maternal fasting. A small but consistently negative $\mathrm{A}-\mathrm{V}$ difference of the branched-chain $\alpha$-keto acids is also demonstrated. The output of the $\alpha$-keto acids averaged less than $10 \%$ of the simultaneous uptake of the corresponding amino acid as determined by simultaneously measured A-V differences. We interpret this finding to indicate that the branched-chain carbon skeleton not used for protein synthesis is degraded, at least through the initial decarboxylation, within the fetal hindlimb.
The ratio of the ketoacid $A-V$ difference to amino acid $A-V$ difference does not increase during fasting. This implies sufficient dehydrogenase activity within the fetal hindlimb to account for nearly all the flux through the transaminase during the fasted state.

Any use of the amino acids other than for protein synthesis would require export of nitrogen out of the hindlimb. During maternal fasting, $\alpha$-amino nitrogen is exported from fetal hindlimb as alanine and glutamine, in direct contrast to the uptake of these amino acids when the ewe is fed. The negative molar A$\mathrm{V}$ concentration differences for nitrogen leaving the hindlimb as alanine and glutamine in the fasted state almost exactly equals the positive molar A-V concentration difference of nitrogen entering as branched-chain amino acids (Fig. 2). Although free ammonia, another potential form in which nitrogen may be transported, was not measured in the present study, we have previously demonstrated a persistent A-V difference for ammonia, the magnitude of which is not affected by fasting (6).

The results of several studies have indicated that the peripheral tissues of ruminant animals may not utilize branched-chain amino acids to any significant extent. In this regard, Ballard et al. (15) demonstrated insignificant A-V differences of the branched-chain amino acids in fed nonpregnant adult sheep, and an efflux of considerable proportion of all three branched-chain amino acids in the fasted state. Heitman et al. (16) have shown a consistent output of the branched-chain amino acids by hindlimb and kidney, with simultaneous uptake by liver, which was accentuated by fasting. Ahmed et al. (17) also demonstrated output of branched-chain amino acids by the hind limb of steers when fasted. Coward and Buttery (18), using a perfused sheep hemidiaphragm preparation, demonstrated very small amounts of the branched-chain amino acids to be metabolized within the sheep myocyte.

These data have been interpreted to suggest that the ruminant may have lesser skeletal muscle enzyme activity for the metabolism of the branched-chain amino acids than do nonruminants. Busboom et al. (19) have shown that branched-chain amino acid transaminase activity is low in the skeletal muscle of wether lambs and decreases with fasting. Similar trends, but lower total activity, were seen for branched-chain keto acid dehydrogenase activity. These findings are in contrast to similar studies in nonruminants, which generally have been shown to have an increase in the branched chain amino acid transaminase and branched chain keto acid dehydrogenase activities in skeletal muscle during fasting (20). In Busboom's study, adipose tissue had 100-fold higher activity for both enzymes; the activity in adipose tissue also decreased with fasting.

In contrast to the data obtained from adult ruminants, the results of the present study support an ovine fetal model for peripheral branched-chain amino acid metabolism similar to that proposed for nonruminant animals (21-24). In this model, peripheral tissues, especially skeletal muscle, appear to be the major site of branched-chain amino acid transaminase activity,

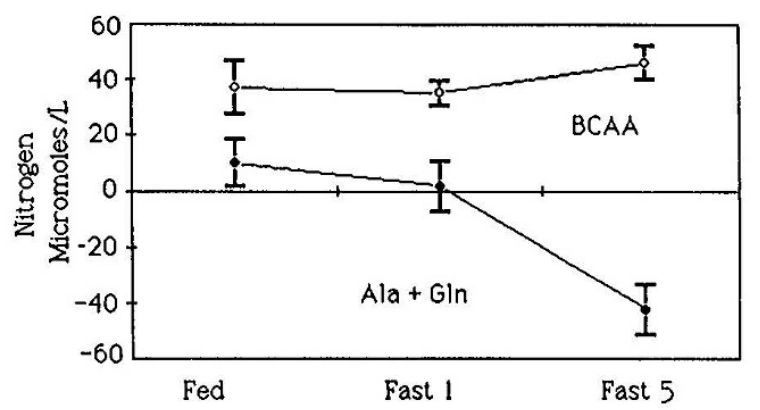

Fig. 2. Effect of maternal nutritional state on fetal hindlimb nitrogen A-V difference as branched-chain amino acids or alanine + glutamine. Each point represents mean \pm SEM for all animals $(n=8)$. 
thus allowing these amino acids to bypass uptake by the liver, to be taken up by skeletal muscle instead. During fasting this process becomes more efficient, primarily because of an increase in muscle BCAAT activity. Transport of branched-chain amino acid nitrogen out of the periphery to the liver is in the form of alanine and glutamine. While the carbon for alanine synthesis is thought to be derived from pyruvate, glutamine carbon may be derived from the carbon skeleton of valine and isoleucine. In this manner, by contributing net gluconeogenic precursor to the liver, branched-chain amino acids may participate in the overall glucose economy of the undernourished fetus.

The results of the present study clearly underscore the importance of the branched-chain amino acids to the overall metabolism of the ovine fetal hindlimb, and suggest that at least during fetal life, ruminant peripheral tissues possess ample enzymatic activity to utilize these amino acids. As an energy source, uptake of these three amino acids could account for $20 \%$ of total hindlimb oxygen uptake during the fed state; during fasting, when reliance on glucose as an energy source falls precipitously, this increases to $55 \%$, even when loss of glutamine carbon is taken into account. That the branched-chain carbn skeletons are utilized within the anatomic hindlimb is evidenced by the negligible venous efflux of the corresponding $\alpha$-keto acid. The precise site of the activity, whether myocyte, adipocyte, or some other tissue type within the hindlimb, remains to be demonstrated. In addition to use for protein synthesis, the carbon skeleton of these amino acids may be used as an oxidative fuel, as substrate for lipid synthesis in the case of leucine, and valine and isoleucine may be used for glutamine synthesis.

Acknowledgments. The authors acknowledge the expert assistance of D. Wood, M. Wass, G. H. Robinson, and P. Nicholson.

\section{REFERENCES}

I. Battaglia FC, Meschia G 1978 Principle substrates of fetal metabolism. Physiol Rev 58:499-527

2. Hay WW, Sparks JW, Wilkening RB, Battaglia FC, Meschia G 1984 Fetal glucose uptake and utilization as functions of maternal glucose concentration. Am J Physiol 256:E237-E242

3. Sparks JW, Hay WW. Bonds D. Meschia G, Battaglia FC 1982 Simultaneous measurement of lactate turnover rate and umbilical lactate uptake in the fetal lamb. J Clin Invest 70:179-192
4. Gresham EL, James EJ, Raye JR, Battaglia FC, Makowski EL, Meschia G 1972 Production and excretion of urea by the fetal lamb. Pediatrics 50:372379

5. Lemons JA, Schreiner RL 1983 Amino acid metabolism in the ovine fetus. Am J Physiol 244:E459-466

6. Liechty EA, Lemons JA 1984 Changes in ovine fetal hindlimb metabolism during maternal fasting. Am J Physiol 246:E430-435

7. Raabo E, Terkildsen TC 1960 On the enzymatic determination of blood glucose. Scand J Clin Invest 12:402

8. Marbach EP, Weill MH 1967 Rapid enzymatic measurement of blood lactate and pyruvate. Clin Chem 13:314

9. Abumrad NN, Wise KL, Williams PE, Abamrad NA, Lacy WW Disposal of alpha-ketoisocaproate: roles of liver, gut, and kidneys. Am J Physiol 243:E123-E131

10. Sokal RR, Rohlf FJ 1981 Single classification analysis of variance. In: Biometry the Principles and Practice of Statistics in Biological Research. WH Freeman and Company. San Francisco, pp 244-245

11. Einot I, Gabriel KR 1975 A study of the powers of several methods of multiple comparisons. J Am Statist Assoc 70:574-583

12. Elia M, Neale G, Livesey G 1985 Alanine and glutamine release from the human forearm: effects of glucose administration. Clin Sci 69:123-133

13. Garber AJ, Karl IE, Kipnis DM 1976 Alanine and glutamine synthesis and release from skeletal muscle II. The precursor role of amino acids in alanine and glutamine synthesis. J Biol Chem 251:836-843

14. Singh SS, Sparks JW, Meschia G, Battaglia FC, Makowski EL 1984 Comparison of fetal and maternal hind limb metabolic quotients in sheep. Am J Obstet Gynecol 149:441

15. Ballard FJ, Filsell OH, Jarret IG 1976 Amino acid uptake and output by the sheep hindlimb. Metabolism 25:415-418

16. Heitman RN, Bergen WG, Ames NK 1980 Integration of amino acid metabolism in sheep: effects of fasting and acidosis. Am J Physiol 239:E248-254

17. Ahmed BM, Bergen WG, Ames NK 1983 Effect of nutritional state and insulin on hindlimb amino acid metabolism in steers. J Nutr 113:1529-1543

18. Coward BJ, Buttery PJ 1982 Metabolism of the perfused ruminant muscle. $J$ Agric Sci Camb 98:307-316

19. Busboom JR, Merkel A, Bergen WG 1983 The effects of dietary protein content and fasting on tissue leucine transaminase and alpha-ketoisocaproate dehydrogenase in wethers. J Animal Sci 57:(suppl 1):189(abstr)

20. Paul HS, Adibi SA 1984 Regulation of branched chain amino acid catabolism. In: Adibi SA, Fekl W, Langenbeck U, Schauder P (eds) Regulation of Branched Chain Amino Acid Catabolism in Branched Chain Amino and Keto Acids in Health and Disease, Karger, Basel, pp 182-219

21. Abumrad NN, Robinson RP, Gooch BR, Lacy WW 1982 The effect of leucine infusion on substrate flux across the human forearm. J Surg Res 32:453463

22. Davis EJ, Lee SEC 1985 Amino acid metabolism by the perfused rat hindquarter. Biochem J 229:19-29

23. Wagenmakers AJM, Veerkemp JH 1984 The effect of starvation on branched chain 2-oxoacid oxidation in rat muscle. Biochem J 219:253-260

24. Haymond MW, Miles JM 1982 Branched chain amino acids as a major source of alanine nitrogen in man. Diabetes 31:86-89 